\title{
Cold ultrarelativistic pulsar winds as potential sources of galactic gamma-ray lines above $100 \mathrm{GeV}$
}

\author{
F. Aharonian ${ }^{1,2}$, D. Khangulyan ${ }^{3}$, and D. Malyshev ${ }^{4}$
}

\author{
1 Dublin Institute for Advanced Studies, 31 Fitzwiliam Place, Dublin 2, Ireland \\ e-mail: Felix. Aharonian@mpi-hd.mpg.de \\ 2 Max-Planck-Institut fur Kernphysik, Postfach 103980, 69029 Heidelberg, Germany \\ ${ }^{3}$ Institute of Space and Astronautical Science/JAXA, 3-1-1 Yoshinodai, Chuo-ku, Sagamihara, 252-5210 Kanagawa, Japan \\ ${ }^{4}$ Bogolyubov Institute for Theoretical Physics, Metrologichna str. 14-b, 03680 Kiev, Ukraine
}

Received 24 July 2012 / Accepted 30 September 2012

\section{ABSTRACT}

\begin{abstract}
Context. The evidence of line-like spectral features above $100 \mathrm{GeV}$, in particular at $130 \mathrm{GeV}$, which have been recently reported from some parts of the Galactic plane, poses serious challenges for any interpretation of this surprise discovery. It is generally believed that the unusually narrow profile of the spectral line cannot be explained by conventional processes in astrophysical objects, and, if real, is likely to be associated with dark matter.

Aims. In this paper we argue that cold ultrarelativistic pulsar winds can be alternative sources of very narrow gamma-ray lines. Methods. We demonstrate that Comptonization of a cold ultrarelativistic electron-positron pulsar wind in the deep Klein-Nishina regime can readily provide very narrow $(\Delta E / E \leq 0.2)$ distinct gamma-ray line features. To verify this prediction, we produced photon-count maps based on the Fermi LAT data in the energy interval 100 to $140 \mathrm{GeV}$.

Results. We confirm earlier reports of the presence of marginal gamma-ray line-like signals from three regions of the Galactic plane. Although the maps show some structure inside these regions, unfortunately the limited photon statistics do not allow any firm conclusion in this regard.

Conclusions. The confirmation of $130 \mathrm{GeV}$ line emission by low-energy threshold atmospheric Cherenkov telescope systems, in particular by the new $28 \mathrm{~m}$ diameter dish of the H.E.S.S. array, would be crucial for resolving the spatial structure of the reported hotspots, and thus for distinguishing between the dark matter and pulsar origins of the "Fermi Lines".
\end{abstract}

Key words. radiation mechanisms: non-thermal - methods: analytical - pulsars: general - gamma rays: diffuse background

\section{Introduction}

Recent reports of a possible narrow line-like feature in the spectrum of gamma-ray emission at $130 \mathrm{GeV}$ from the Galactic center region, and, presumably, from some other parts of the Galactic plane (Bringmann et al. 2012; Weniger 2012; Tempel et al. 2012; Boyarsky et al. 2012; Su \& Finkbeiner 2012), have received a prompt and enthusiastic reaction from the astrophysics and particle physics communities. Despite the marginal statistical significance of the reported signals and some outstanding questions and inconsistencies, the implications of these results are hotly debated, basically in the context of dark matter (DM). This is motivated not only by the recognition of the potential of gamma-ray observations for indirect searches of DM (for a recent review see Bergström 2012) and the overall excitement caused by the possible association of the gamma-ray line at $130 \mathrm{GeV}$ with DM (see e.g. Buckley \& Hooper 2012), but also by the unusual characteristics of the signal.

It is generally believed that the width of the $130 \mathrm{GeV}$ line (of a few tens of $\mathrm{GeV}$ ) is too narrow to be explained by any physical process except for annihilation of $\mathrm{DM}^{1}$. On the other hand, some other features - in particular the significant shift

\footnotetext{
1 Based on the analysis of Fermi LAT data by Weniger (2012), it has been argued (Profumo \& Linden 2012) that the contamination of a bright featureless power-law background component (related e.g. to the radiation of the interstellar medium) by hard photons arriving from Fermi bubbles with a sharp spectral break between 100 and $150 \mathrm{GeV}$, can mimic a spurious line. However, a closer look at the morphology
}

in the center of gravity of the signal from the position of the Galactic center (Su \& Finkbeiner 2012), as well as the possible presence of gamma-ray lines at other energies (Boyarsky et al. 2012) and from other parts of the Galactic plane (Tempel et al. 2012; Boyarsky et al. 2012) - challenge the DM origin of the reported signal. Unfortunately, the marginal gamma-ray photon statistics do not allow definite conclusions in this regard.

The situation will be somewhat improved after doubling the photon statistics above $100 \mathrm{GeV}$ by observations of the Galactic plane with the Fermi Large Area Telescope (LAT) over the next several years. The development of new dedicated approaches to the data reduction, which are focused on the highest energy domain of Fermi LAT, may also help clarify some of the current uncertainties and inconsistencies. Finally, there is hope that soon the low-energy threshold imaging Cherenkov telescopes, in particular the new $28 \mathrm{~m}$ diameter dish of the H.E.S.S. telescope array located in the southern hemisphere (see http://wWw . mpi-hd.mpg.de/hfm/HESS/), will contribute strongly to clarifying the question of the origin of $\geq 100 \mathrm{GeV}$ gamma-ray line(s) - are they instrumental, cosmological (DM), or astrophysical?

The last option implies production of gamma-rays by accelerated particles. So far it has been discarded, basically because of the common belief that conventional high-energy processes with involvement of ultrarelativistic particles could not reproduce gamma-ray line features. In Sect. 2 we briefly discuss different gamma-ray production mechanisms in the very-high energy

shows that the $130 \mathrm{GeV}$ feature is most likely not associated with Fermi Bubbles (see e.g. Tempel et al. 2012). 
(VHE) domain in the context of their ability to produce sharp gamma-ray lines, and argue that the inverse Compton scattering in the Klein-Nishina regime by cold ultrarelativistic electronpositron pulsar winds can result in sharp gamma-ray line emission. The conditions for reproducing narrow Klein-Nishina line profiles are discussed in Sect. 3. Finally, in Sect. 4 we describe our study of the spatial distribution of $E \geq 100 \mathrm{GeV}$ photons based on the Fermi LAT data. We confirm the results reported earlier on the presence of marginal gamma-ray line-like signals from three regions of the Galactic plane, but because of limited photon statistics, we cannot make a strong statement on the existence of localized hot spots inside the "Fermi Line" regions. The results and conclusions are summarized in Sect. 5.

\section{Astrophysical VHE gamma-ray lines?}

Interactions of relativistic particles with a broad energy distribution cannot result in sharp gamma-ray spectral features. Moreover, even in the case of interactions of monoenergetic relativistic particles, the energy spectra of resulting gamma-rays generally are broad. For example, a $1 \mathrm{TeV}$ proton interacting with surrounding gas produces gamma-rays (through production and decay of $\pi^{0}$-mesons) with an average energy of $100 \mathrm{GeV}$, but the distribution of gamma-rays is very broad with $\Delta E / E \gg 1$ (see e.g. Kelner et al. 2006). Actually, this is true for all hadronic interactions, including photomeson processes, with the involvement of secondary $\pi^{0}$-mesons.

Narrow gamma-ray distributions can be expected from monoenergetic beams of ultrarelativistic heavy ions, e.g. ${ }^{56} \mathrm{Fe}$, excited at interactions with surrounding low-frequency radiation. The Doppler-boosted gamma-ray emission due to the deexcitation of these nuclei with Lorentz factor $\Gamma \geq 10^{5}$ may lead, in principle, to a rather narrow lines at energy $E=\Gamma E^{*} \sim$ $100 \mathrm{GeV}$ (typically the prompt de-excitation gamma-ray lines are produced with energy in the frame of the nucleus $E^{*} \sim$ $1 \mathrm{MeV})$. However, the efficiency of this mechanism in typical astrophysical environments is very low (Aharonian \& Taylor 2010). Also, the disintegration of the primary nuclei would lead to emission of a large number of lines from secondary nuclei, and eventually to a rather broad distribution.

The synchrotron radiation of charged particles, electrons or protons, also leads to broad-band emission. Even in the case of the radiation of monoenergetic particles in a homogeneous magnetic field, the spectral energy distribution (SED) is broad, $E^{2} \mathrm{~d} N / \mathrm{d} E \propto E^{4 / 3} \exp \left(-E / E_{0}\right)$ with $\Delta E / E \geq 1$. In addition to synchrotron radiation, there are two other processes for producing high-energy gamma-rays by relativistic electrons - bremsstrahlung and inverse Compton scattering (IC). Bremsstrahlung of monoenergetic electrons results in very hard, but still continuous gamma-ray distribution, $E^{2} \mathrm{~d} N / \mathrm{d} E \propto E$. One should also mention that at very high energies the electron cooling typically is dominated by synchrotron and IC radiation losses, thus bremsstrahlung plays an important role only at relatively low, sub-GeV energies.

In contrast, the inverse Compton scattering of relativistic electrons is a universal gamma-ray production mechanism that can work with very high efficiency throughout the entire gammaray domain from $\mathrm{MeV}$ to $\mathrm{TeV}$ energies in diverse astrophysical environments from compact objects like pulsars and AGN to extended sources like supernova remnants and clusters of galaxies (see e.g. Aharonian 2004). In the Thompson regime, IC scattering boosts the energy of target photons to $E_{\gamma} \propto \varepsilon \Gamma^{2}$, where $\varepsilon$ is the target photon energy, and $\Gamma$ the electron Lorentz factor. Thus, to produce a narrow gamma-ray spectral feature, one should require very narrow distributions for both relativistic electrons and target photons. However, even in this case, the distribution of the upscattered radiation is relatively broad due to the character of the cross-section in the Thompson regime. Another principal limitation comes from the requirement of narrowness of the target photon field. Even in the case of blackbody radiation, the width of the photon distribution exceeds the average photon energy $\varepsilon \approx 3 \mathrm{kT}$, thus the original distribution of target photons will be shifted to higher energies by a factor of $\Gamma^{2}$, and non-negligibly broaden due to the cross-section in the Thompson regime. Even in the case of an abrupt cutoff in the electron spectrum and the Planckian distribution of target photons, the spectrum of gamma-rays contains a rather long exponential tail (Lefa et al. 2012).

The picture changes dramatically, however, when the IC scattering proceeds in the Klein-Nishina regime, i.e. when $b=\varepsilon E_{\mathrm{e}} / m_{\mathrm{e}}^{2} c^{4} \geq 1$. In this case, the target photons take the entire momentum of the electron. Thus, independent of the energy distribution of target photons, one may expect a line shape for the upscattered radiation if the electrons have a very narrow distribution. In this regard, the narrowness of the energy distribution of electrons becomes the most demanding condition for forming sharp gamma-ray lines. Obviously, the width of the electron distribution cannot exceed the width of the claimed $130 \mathrm{GeV}$ gamma-ray line, $\Delta E / E \ll 1$, so even a Maxwellian type distribution of electrons cannot satisfy this condition; one needs almost monoenergetic electrons to reproduce a very narrow gamma-ray line. While such a distribution can hardly be formed in standard particle acceleration scenarios with the involvement of stochastic processes, monoenergetic beams of relativistic particles in astrophysics are possible. They can be realized in the form of bulk motion of hydrodynamic flows, in particular in the so-called cold ultrarelativistic electron-positron winds of pulsars.

The generally accepted paradigm of pulsars and their synchrotron nebulae (Rees \& Gunn 1974; Kennel \& Coroniti 1984) postulates the existence of an intermediate substance with quite unusual properties - a cold ultrarelativistic electron-positron wind which originates in the pulsar's magnetosphere and terminates in the interstellar medium. The electric fields in the magnetosphere accelerate electrons, which radiate photons with energy high enough to interact with the surrounding electromagnetic field. This initiates electromagnetic cascades, leading to the formation of a relativistic outflow of a dense electron-positron plasma - the wind. Initially, the rotational energy losses are released in the form of Poynting flux (electromagnetic energy), making wind's Lorentz factor relatively modest. The conversion of the Poynting flux leads to the "acceleration" of the wind with a Lorentz factor as large as $\Gamma_{\mathrm{w}} \sim 10^{5}$. Obviously, in this case all electrons move with the same (bulk motion) speed.

In cold winds, electrons move together with magnetic field and, therefore, do not emit synchrotron radiation. Nevertheless, they are (potentially) visible owing to the characteristic inverse Compton radiation of the ultrarelativistic electrons of the wind (Bogovalov \& Aharonian 2000). For isolated pulsars, a sharp gamma-ray line can be produced at Comptonization of the electron-positron wind by thermal X-rays arriving from the surface of the neutron star. The mechanism can be effective only if the conversion of the Poynting flux to kinetic energy of the bulk motion ("acceleration" of the wind) takes place close to the light cylinder (Bogovalov \& Aharonian 2000). In the case of pulsars with bright nonthermal broad-band magnetospheric emission, e.g. in the Crab pulsar, the major fraction of the wind energy is channeled into the pulsed VHE continuum, but not into 
the line. It is likely (Aharonian et al. 2012) that this component of radiation of the pulsar wind is responsible for the recently detected VHE pulsed radiation of the Crab pulsar (VERITAS Collaboration et al. 2011; Aleksić et al. 2012).

Gamma-ray line-like structures are expected also from binary systems containing a pulsar and a luminous optical star (Ball \& Kirk 2000; Khangulyan et al. 2007). In this case the efficiency of IC scattering is higher because of the presence of copious target photons provided by the companion star, but the gamma-ray lines are less sharp than for the isolated pulsars because of lower values of the Klein-Nishina parameter $b$. It has recently been argued (Khangulyan et al. 2012) that the bright flare of the binary pulsar PSR B1259-63/LS2883, detected by Fermi in 2011 (Abdo et al. 2011; Tam et al. 2011), could be best explained by the IC scattering of the unshocked pulsar. The spectral measurements of Fermi LAT require a relatively modest Lorentz factor of the wind, $\Gamma_{\mathrm{w}} \approx 10^{4}$. The IC scattering proceeds in the Thompson regime, therefore the resulting gammaradiation has a rather broad energy distribution (Khangulyan et al. 2012).

Finally, one should mention two other (highly speculative) scenarios of formation of a very narrow multi-GeV spectral gamma-ray line feature. It could be related to the $0.511 \mathrm{MeV}$ line produced at annihilation of $\left(\mathrm{e}^{+} \mathrm{e}^{-}\right)$pairs in cold plasma of an outflow (jet or a wind) relativistically moving with Lorentz factor $\Gamma \geq 10^{4}$. A very sharp rise in the gamma-ray spectrum can also be expected in a very different scenario as due to the process of photon-photon absorption in optically thick gamma-ray sources.

\section{Formation of sharp Klein-Nishina lines}

The strongest argument in favor of a nonastrophysical origin of the $\sim 130 \mathrm{GeV}$ spectral feature is its very sharp profile. It is very narrow, $\Delta E / E \approx \frac{20 \mathrm{GeV}}{130 \mathrm{GeV}} \simeq 15 \%$, with an exponential rise and decay of the flux (which corresponds to linear dependence as seen in the semilogarithmic scale plots), while we expect much smoother spectral distributions in typical astrophysical situations. Nevertheless, in the case of inverse Compton scattering, such profiles can be reproduced by monoenergetic electrons provided that the scattering proceeds in the deep Klein-Nishina regime. In this case the shape of the radiation spectrum is fully determined by a single parameter, $b=4 \omega \Gamma$, where $\omega=\varepsilon / m_{\mathrm{e}} c^{2}$ and $\Gamma=E_{\mathrm{e}} / m_{\mathrm{e}} c^{2}$ are the energy of the target photon in $m_{\mathrm{e}} c^{2}$ units and the electron Lorentz factor, respectively. In Fig. 1 IC gamma-ray spectra are shown calculated for four different values of the parameter $b: 1,7,50$, and 100 . The filled regions below each line correspond to the intervals where the flux level exceeds $50 \%$ of the maximum value. Since the reported gammaray line flux is above the background only by a factor of two, for characterization of the line profile we use the half-height width as a measure of the line thickness. This also allows us to define the hardness of the left wing of the line by the power-law slope at the point where the flux level reaches $50 \%$ of the maximum value (the lower energy point). The line thickness and hardness determined in this way are shown in Fig. 2 as functions of the parameter $b$.

In the Klein-Nishina regime, the upscattered photons repeat, to a large extent, the spectrum of parent electrons. Therefore even the Maxwellian distribution of electrons, which is generally considered as a very narrow one in the context of particle acceleration scenarios and which can be realized only with very specific conditions, cannot explain the reported very narrow line.

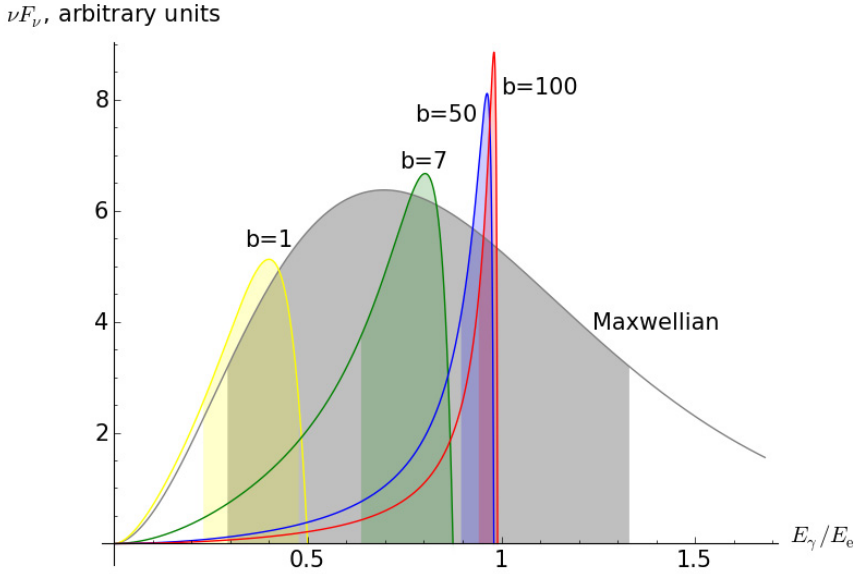

Fig. 1. Color: energy spectra of the inverse Compton radiation of monoenergetic electrons upscattering isotropic target photons for 4 different values of the parameter $b: 1,7,50$, and 100 . The energy of gammarays is in units of the electron energy. Gray: the gamma-ray spectrum produced by electrons with relativistic Maxwellian distribution; in this case, the photon energies are in units of $4 \Theta$, where $\Theta$ is the "temperature" of Maxwellian distribution.

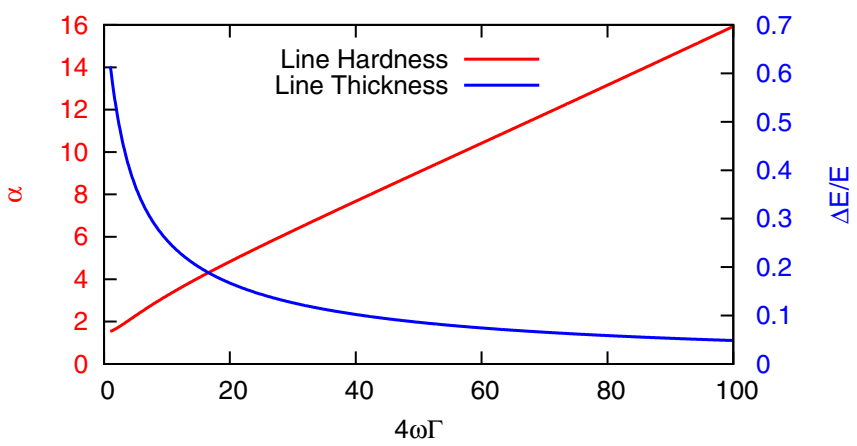

Fig. 2. The thickness and hardness of the Klein-Nishina line as functions of the $b$-parameter.

This is illustrated in Fig. 1, where the IC gamma-ray spectrum produced by electrons with a Maxwellian distribution, $\mathrm{d} N_{\mathrm{e}} / \mathrm{d} \Gamma \propto$ $\Gamma^{2} \exp (-\Gamma / \Theta)$ is shown; there, the energy of gamma rays are expressed in units of $4 \Theta$, where $\Theta$ is the electron "temperature". It can be seen that the half-height width of this spectrum is comparable to the energy at which the distribution maximum is achieved at $E_{\gamma} \sim 2.8 \Theta$, and hence much broader than the observed one. Meanwhile, the results presented in Fig. 1 show that the monoenergetic distribution of electrons does provide very sharp feature at $E_{\gamma}=E_{\mathrm{e}}$ with a width $\Delta E / E \leq 15 \%$, provided that the Klein-Nishina parameter $b \geq 30$. The corresponding hardness of the line, $\alpha=6$, also is in good agreement with observations. The fixed value of the parameter $b$ implies a monoenergetic distribution of target photons. Although in specific calculations one should assume a more realistic spectrum of seed photons, this cannot significantly change the conclusions as long the parameter $b$ remains large. For $b \gg 1$, we can adopt with good accuracy $E_{\gamma}=E_{\mathrm{e}}$. Then, one can immediately constrain the energy range of target photons:

$\varepsilon \geq 15\left(E_{\gamma} / 130 \mathrm{GeV}\right)^{-1} \mathrm{eV}$,

where $E_{\gamma}$ is the energy of the detected line. In the case of Planckian radiation field, the above photon energy corresponds approximately to the radiation temperature of $\sim 5 \times 10^{4} \mathrm{~K}$. In 

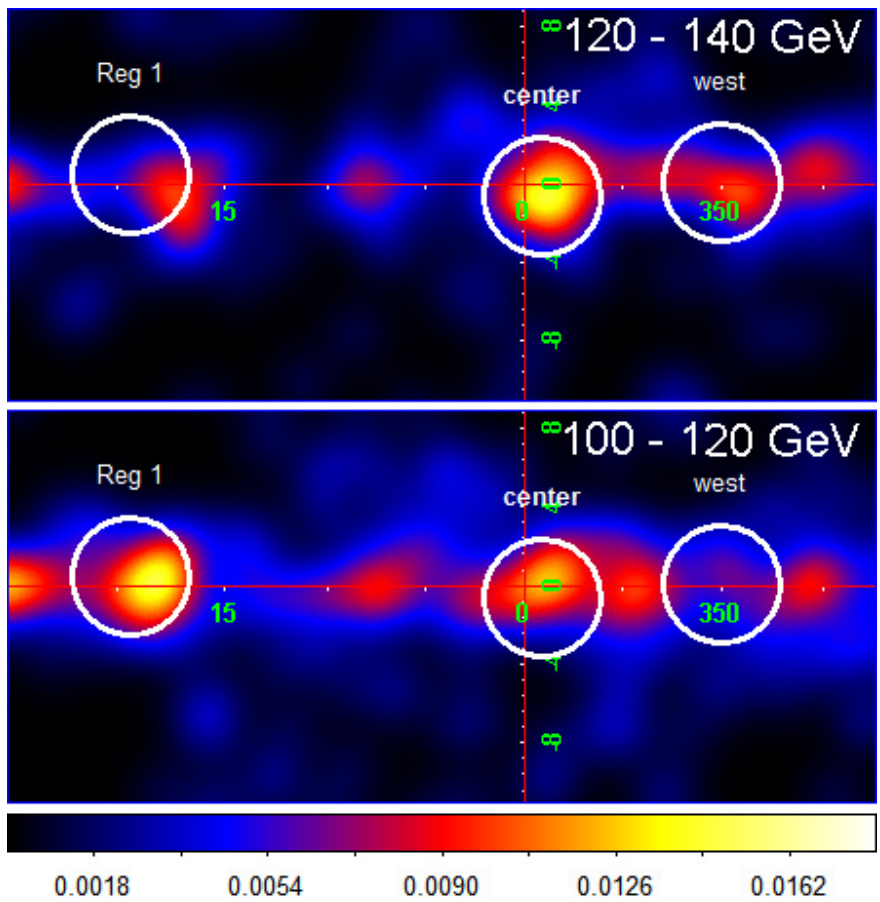

Fig. 3. The count maps of the "Central", "West" and Reg 1 regions (see the text) in two energy intervals, $100-120 \mathrm{TeV}$ and $120-140 \mathrm{TeV}$, smoothed with a $3^{\circ}$ FWHM Gaussian kernel

the case of isolated pulsars, thermal emission from the surface of the neutron star would be the main source of seed photons for the formation of gamma-ray lines. The neutron star's surface temperature exceeds this limit by two orders of magnitude, thus from isolated pulsars we may expect extremely narrow gamma-ray lines. In binary systems with pulsars, the seed photons are provided by companion stars with radiation temperatures slightly less than the above estimate. Correspondingly, the gamma-ray lines would be broader with $\Delta E / E \geq 20 \%$; this still marginally agrees with observations of the $130 \mathrm{GeV}$ line from the Galactic center region. On the other hand, the efficiency of formation of Klein-Nishina lines from pulsar winds in binary systems is significantly higher in binary systems than in isolated pulsars.

\section{Structure of gamma-ray line "hotspots"}

An astrophysical origin of the $130 \mathrm{GeV}$ line, in general, and, more specifically, its association with pulsars, would imply that the extended regions of excess emission are not truly diffuse structures, but rather represent superpositions of multiple unresolved discrete gamma-ray sources. To verify this hypothesis we analyzed 52-month data of Fermi/LAT using the latest Fermi software package v9r27p1. The LAT aboard the Fermi satellite is a pair-conversion gamma-ray detector operating between $20 \mathrm{MeV}$ and $300 \mathrm{GeV}$. The Fermi LAT has a wide field of view of $\sim 2.4 \mathrm{sr}$ at $1 \mathrm{GeV}$, and observes the entire sky every two orbits. The details on this instrument can be found in Atwood et al. (2009).

Here we consider three regions from which excess gammaray lines have been reported (Tempel et al. 2012; Su \& Finkbeiner 2012; Boyarsky et al. 2012) in the energy interval between 100-140 GeV. The results are presented in Figs. 3 and 4, and summarized in Table 1.
Table 1. Coordinates and names of considered regions and point-like hot spots detected in these regions.

\begin{tabular}{lccccc}
\hline \hline Region & Hot spot & $l$ & $b$ & $R$, deg & $T S$ \\
\hline Central & & 359.0 & -0.7 & 3 & \\
& 2FGL J1745.6-2858 & 359.97 & -0.04 & 0.02 & 8.5 \\
& 2FGL J1740.4-3054c & 357.73 & -0.08 & 0.16 & 8.4 \\
& $\mathrm{c} 1$ & 359.02 & -1.41 & 0.08 & 7.5 \\
& $\mathrm{c} 2$ & 358.45 & -1.08 & 0.12 & 9.2 \\
& $\mathrm{c} 3$ & 358.43 & 1.47 & 0.23 & 8.6 \\
\hline West & & 350.0 & 0.0 & 3 & \\
& $\mathrm{w} 1$ & 349.55 & -0.57 & 0.19 & 6.9 \\
\hline Reg 1 & & 19.38 & 0.40 & 3 & \\
\hline
\end{tabular}

Notes. $l$ and $b$ are Galactic coordinates of the region (new source), $R-$ the radius of the region (the radius of error-circle of the source). TS is the test-statistical value of the point-like hot spot (if $>5$ ). The significance of the hot spot can be estimated as $\sqrt{T S} \sigma$ (see e.g. Mattox et al. 1996).

In Fig. 3 we show the count maps smoothed with a $3^{\circ}$ FWHM Gaussian kernel in two energy intervals, $100-120 \mathrm{GeV}$ and $120-140 \mathrm{GeV}$. They are consistent with the previously reported results. The locations of the "Central" and "West" regions with excess emission at $120-140 \mathrm{GeV}$ (Tempel et al. 2012) and the "Reg 1" region with the excess emission at $100-120 \mathrm{GeV}$ (Boyarsky et al. 2012) are shown with white circles.

To explore the spatial structures of these regions we produced corresponding count maps smoothed with a $0.25^{\circ}$ Gaussian kernel (comparable to the Fermi PSF at $100 \mathrm{GeV}$ ). The corresponding maps are shown in Fig. 4. The regions from Tempel et al. (2012) and Boyarsky et al. (2012) are shown with white circles. The green crosses show positions of some of the GeV gamma-ray sources from the Fermi two-year source catalog.

In the "Central" region, one can see several "hot spots" (see Table 1 for the coordinates and the test statistical (TS) values of these "hot spots" with $T S \geq 5$ ), however the photon statistics in each of them is limited. The number of photons in five "hot spots" is 16 (from 32 total photons in the region), and the residual flux after removing the excesses is comparable to the background (see e.g. Boyarsky et al. 2012). Thus, the tendency to clumped distribution of photons inside the "Central" region can only be taken as a hint of the presence of discrete sources for VHE gamma-rays. It is interesting to note that the chance of a false-positive detection for the two brightest clumps (7 photons in total) over the uniform photon distribution is about $7 \%$. Their possible association with two hypothetical pulsars seems a very intriguing option in the context of interpreting the possible two-peak structure of the $130 \mathrm{GeV}$ line (Su \& Finkbeiner 2012) as a superposition of Klein-Nisinia lines from two pulsar winds with different Lorentz factors. The search for candidate pulsars at these positions would be a straightforward test for verifying this hypothesis, although the detection of pulsars through their magnetospheric emission cannot be guaranteed because of possible misorientations of their radiation cones.

The "West" (at 120-140 GeV) and "Reg 1" (at 100-120 GeV) regions look more diffuse in gamma-rays (see Fig. 4, middle and right panels), however the poor photon statistics do not allow any conclusion concerning the level of clumpiness in these regions. While to confirm gamma-ray line signals from these regions an increase in photon statistics by a factor of two or three would be adequate, the study of spatial 


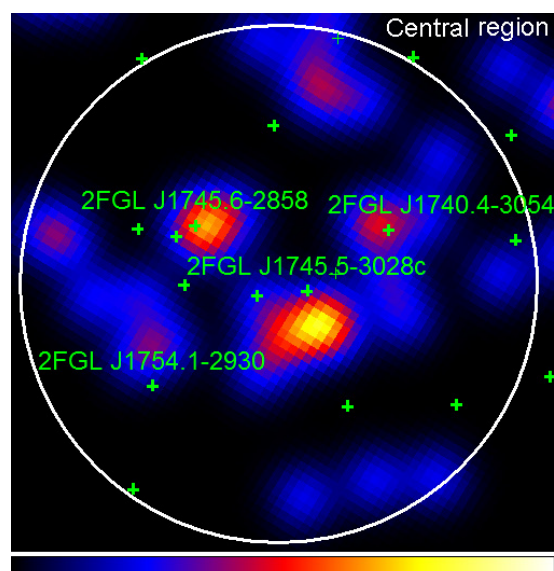

$\begin{array}{lllllllll}0.01 & 0.02 & 0.03 & 0.04 & 0.05 & 0.06 & 0.07 & 0.08 & 0.09\end{array}$

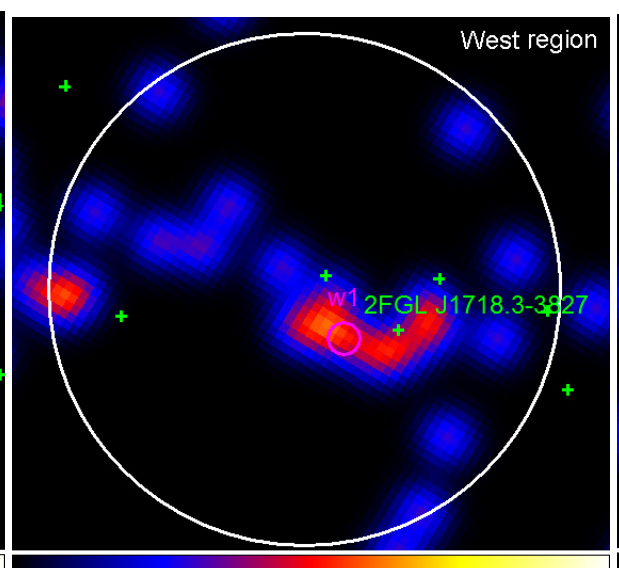

$\begin{array}{lllllllll}0.01 & 0.02 & 0.03 & 0.04 & 0.05 & 0.06 & 0.07 & 0.08 & 0.09\end{array}$

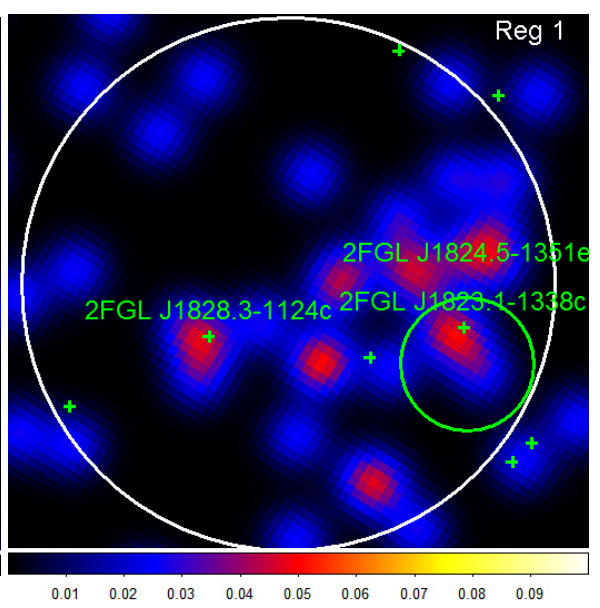

Fig. 4. Photon-count maps of the "Central" (left) and "West" (middle) regions at 120-140 GeV and the "Reg 1" region at 100-120 GeV energy bands smoothed with a $0.25^{\circ}$ Gaussian kernel ( $\approx 95 \%$ Fermi PSF at $100 \mathrm{GeV}$ ). The corresponding regions from Tempel et al. (2012) and Boyarsky et al. (2012) are shown with white circles, while the sources from the Fermi LAT two-year catalog are shown with green crosses.

distributions of gamma-rays inside these regions is a more demanding task that requires much higher photon statistics.

\section{Summary}

In this paper we argued that there is a viable alternative to the $\mathrm{DM}$ origin of the $130 \mathrm{GeV}$ gamma-ray line as recently reported in the Galactic gamma-ray emission. We demonstrated that very sharp gamma-ray spectral lines can be produced by pulsar winds through their Comptonization, predominantly by energetic (UV, $\mathrm{X}$-ray) radiation with a relatively narrow spectral distribution, so that the IC scattering proceeds in the deep Klein-Nishina limit. In principle, these conditions can be fulfilled both in isolated pulsars and binary systems.

The current paradigm, which connects pulsars with their synchrotron nebulae through cold ultrarelativistic electron-positron winds, postulates that the electron-positron wind with a Lorentz factor between $10^{4}$ to $10^{6}$ carries away almost the entire rotational energy lost by the pulsar. Thus, under the condition of effective Comptonization of the wind, a substantial fraction of the spin-down luminosity of a pulsar $L_{\text {rot }}$ can be released in a single gamma-ray line. Depending on the intensity of illumination of the pulsar wind by surrounding radiation field(s), the efficiency of formation of such lines can be very high, formally close to $100 \%$.

Interestingly, the narrow profile of the $130 \mathrm{GeV}$ line argues against such an extreme efficiency, which can be realized in the case of an optically thick source. This would imply not only strong attenuation of gamma-rays due to photon-photon pair production, but also significant broadening of the line because of the cooling of electrons. However, both effects become negligible in the case of the still very high, $\leq 10-20 \%$ efficiency of the wind Comptonization. Conservative estimates show that while such an efficiency can be readily achieved in binary systems, in the case of isolated pulsars the efficiency is expected to be very low unless the acceleration of the wind takes place close to the light cylinder.

The efficiency transforming the kinetic energy of the pulsar wind into Klein-Nishina gamma-ray lines is key, but its discussion is beyond the scope of this paper. Clearly, under certain conditions, the efficiency can be quite high, and for pulsars with spin-down luminosities exceeding $10^{36} \mathrm{erg} / \mathrm{s}$ and wind
Lorentz factor $\geq 2 \times 10^{5}$, one may expect $\geq 100 \mathrm{GeV}$ gamma-ray lines with luminosities exceeding the bolometric luminosities of magnetospheric emission of pulsars. Moreover, one cannot exclude other configurations of compact objects, e.g. magnetars, with (hypothetical) relativistic electron-positron winds, as effective multi-GeV gamma-ray emitters.

In this regard a natural question arises: if the pulsars can work as effective generators of VHE gamma-ray lines, why have they not been detected yet? There could be several answers to this question. In particular, in many objects the target radiation field could not be sufficient for effective conversion of the kinetic energy of the wind to IC gamma-radiation. Alternatively, if the wind Lorentz factor is small and/or the target photons have a broad distribution, the IC scattering of electrons in the Thompson regime would lead to a gamma-ray continuum, when the detection and identification could appear to be a difficult task. The formation of the line is only effective for pulsars with wind Lorentz factor exceeding $10^{5}$; the corresponding gamma-ray line is formed around or above $100 \mathrm{GeV}$. At these energies the potential of Fermi LAT is limited because of the small detection area. On the other hand, the current imaging Cherenkov telescopes operate effectively above $100 \mathrm{GeV}$, so could simply have missed the lines around $100 \mathrm{GeV}$. Over the next several years, Fermi LAT can double the photon statistics, which will be sufficient, hopefully, for a firm detection of the $130 \mathrm{GeV}$ line, but still not adequate for morphological studies of the gamma-ray line emitting regions. In this regard forthcoming studies with a new, low-energy threshold, imaging atmospheric Cherenkov telescope systems seem to be more promising. In particular, this goal can be achieved by the new $28 \mathrm{~m}$ diameter dish of the H.E.S.S. telescope array, which is located at a perfect site in the Southern Hemisphere for observations of the Galactic center region. This new instrument with an energy threshold as low as $50 \mathrm{GeV}$, a huge collection area exceeding $10^{4} \mathrm{~m}^{2}$, and energy resolution close to $20 \%$ should allow (in the near future!) deep spectroscopic and morphological studies of the inner galaxy in multi-GeV gamma-ray lines. If confirmed, the existence of such lines may lead to an exciting new research area - Klein-Nishina gamma-ray line astronomy - that will open the way for future ground-based gamma-ray detectors, in particular the Cherenkov Telescope Array (see http://www.cta-observatory.org/) to probe the physics and astrophysics of relativistic outflows, in particular pulsar winds. 
A\&A 547, A114 (2012)

Acknowledgements. We gratefully acknowledge useful conversations with Segey Bogovalov and Roland Crocker. The work of D.M. is supported in part from the SCOPES project IZ73Z0 128040 of Swiss National Science Foundation, grant No CM-203-2012 for young scientists of National Academy of Sciences of Ukraine, Cosmomicrophysics program of the National Academy of Sciences of Ukraine and by the State Program of Implementation of Grid Technology in Ukraine.

\section{References}

Abdo, A. A., Ackermann, M., Ajello, M., et al. 2011, ApJ, 736, L11 Aharonian, F. A. 2004, Very high energy cosmic gamma radiation: a crucial window on the extreme Universe (World Scientific)

Aharonian, F., \& Taylor, A. M. 2010, Astropart. Phys., 34, 258

Aharonian, F. A., Bogovalov, S. V., \& Khangulyan, D. 2012, Nature, 482, 507

Aleksić, J., Alvarez, E. A., Antonelli, L. A., et al. 2012, A\&A, 540, A69

Atwood, W. B., Abdo, A. A., Ackermann, M., et al. 2009, ApJ, 697, 1071

Ball, L., \& Kirk, J. G. 2000, Astropart. Phys., 12, 335

Bergström, L. 2012, Annal. Phys., 524, 479
Bogovalov, S. V., \& Aharonian, F. A. 2000, MNRAS, 313, 504

Boyarsky, A., Malyshev, D., \& Ruchayskiy, O. 2012 [arXiv: 1205.4700]

Bringmann, T., Huang, X., Ibarra, A., Vogl, S., \& Weniger, C. 2012, Cosmol. Astropart. Phys., 7, 54

Buckley, M. R., \& Hooper, D. 2012, Phys. Rev. D, 86, 043524

Kelner, S. R., Aharonian, F. A., \& Bugayov, V. V. 2006, Phys. Rev. D, 74, 034018

Kennel, C. F., \& Coroniti, F. V. 1984, ApJ, 283, 710

Khangulyan, D., Hnatic, S., Aharonian, F., \& Bogovalov, S. 2007, MNRAS, 380 , 320

Khangulyan, D., Aharonian, F. A., Bogovalov, S. V., \& Ribó, M. 2012, ApJ, 752, L17

Lefa, E., Kelner, S. R., \& Aharonian, F. A. 2012, ApJ, 753, 176

Mattox, J. R., Bertsch, D. L., Chiang, J., et al. 1996, ApJ, 461, 396

Profumo, S., \& Linden, T. 2012, Cosmol. Astropart. Phys., 7, 11

Rees, M. J., \& Gunn, J. E. 1974, MNRAS, 167, 1

Su, M., \& Finkbeiner, D. P. 2012 [arXiv: 1207. 7060]

Tam, P. H. T., Huang, R. H. H., Takata, J., et al. 2011, ApJ, 736, L10

Tempel, E., Hektor, A., \& Raidal, M. 2012, Cosmol. Astropart. Phys., 9, 32

VERITAS Collaboration, Aliu, E., Arlen, T., et al. 2011, Science, 334, 69

Weniger, C. 2012, Cosmol. Astropart. Phys., 8, 7 